\title{
Assessing the Role of Minimal Medial Tissue Release during Navigation-Assisted Varus Total Knee Arthroplasty Based on the Degree of Preoperative Varus Deformity
}

\author{
Seikai Toyooka, MD ${ }^{1}$ Hironari Masuda, MD 1 Nobuhiro Nishihara, MD 1 Wataru Miyamoto, MD ${ }^{1}$ \\ Takashi Kobayashi, MD ${ }^{1}$ Hirotaka Kawano, MD ${ }^{1}$ Takumi Nakagawa, MD ${ }^{1}$ \\ ${ }^{1}$ Department of Orthopaedic Surgery, Teikyo University School of \\ Medicine, Tokyo, Japan \\ J Knee Surg 2022;35:1236-1241. \\ Address for correspondence Takumi Nakagawa, MD, Department of \\ Orthopaedic Surgry, Teikyo University School of Medicine, 2-11-1 \\ Kaga, Itabashi-ku, Tokyo 173-8606, Japan \\ (e-mail: takumin-tky@umin.ac.jp).
}

One of the important contribution to good clinical outcomes in total knee arthroplasty (TKA) is adequate soft-tissue balancing that allows neutral joint alignment. ${ }^{1,2}$ Inadequate soft-tissue balancing may lead to complications, such as

received

February 15, 2020

accepted after revision

November 29, 2020

published online

January 20, 2021 instability, abnormal polyethylene wear, aseptic loosening, altered patellofemoral biomechanics, and pain. ${ }^{3-6}$ Excessive release of medial structures, such as the superficial medial collateral ligament (MCL), pes anserinus, posterior oblique (c) 2021. Thieme. All rights reserved. Thieme Medical Publishers, Inc., 333 Seventh Avenue, 18th Floor, New York, NY 10001, USA
DOI https://doi.org/ $10.1055 / \mathrm{s}-0040-1722658$. ISSN 1538-8506. 
ligament (POL), and semimembranosus, may lead to medial instability, midflexion instability, hematoma formation, knee joint elevation, and the need for constrained implants, was contributing to poor postoperative outcomes. ${ }^{7-9} \mathrm{Al}-$ though TKA is successful for many patients, careful management of the medial soft-tissue envelope during surgical exposure, including balanced removal of osteophytes and ligament release, is considered to be important for achieving good postoperative outcomes. ${ }^{10-12}$ Little has been reported about the amount of malalignment that can be corrected adequately with a limited joint line exposure and marginal osteophyte removal during varus deformity correcting TKA.

Following recent advances in computerized navigation technologies, quantitative measures for soft-tissue balancing are currently available, and the real-time identification of the mechanical axis in the knee preoperatively is possible. ${ }^{13,14}$ Real-time data obtained from the navigation system can potentially indicate the need for ligament release and its extent without any recourse to extra bony resection or compromises in alignment. We performed this study to determine the amount of preoperative varus deformity that would be associated with neutral alignment correction after a joint line release, and marginal osteophyte removal had been performed during TKA. We hypothesized that a varus angle greater than 10 degrees would not afford correction with limited joint line release. Understanding the relationships between structural anatomy and response to surgical release may assist preoperative planning and decrease the frequency of extensive medial release during TKA. The purpose of this study was to assess the extent for limited joint line medial soft-tissue release and osteophyte removal to attain manual preresection deformity correction during navigation-assisted TKA procedures.

\section{Materials and Methods}

\section{Study Design and Patients}

A total of 109 patients (131 knees) who had undergone navigation-assisted TKA for advanced knee arthritis at our institution was included. The study protocol was approved by the Institutional Review Board of our institution, and all patients provided informed consent for inclusion in this study. The male-to-female ratio of patients undergoing TKA in our institution differs from the national and global average. ${ }^{15,16}$ To generalize the results of this study to a global standard, the ratio of male-to-female was set at a global standard of $36 \%$ males and $64 \%$ females. Males were included in 47 consecutive knees ( 32 males) that underwent TKA for varus osteoarthritis of the knee from May 2015 to December 2018 , and females were included in 84 consecutive knees (77 females) that underwent TKA for varus osteoarthritis of the knee from May 2015 to May 2017. Patients with osteonecrosis, rheumatoid arthritis, or valgus knee deformity were not enrolled in this study. Preoperative variables were recorded, including age, sex, affected side, hip-kneeankle (HKA) angle, maximum extension, and maximum flexion. The range of motion of the knee was evaluated preoperatively using the goniometer. The degree of arthritis in the operated knees was classified using the KellgrenLawrence (KL) arthritis classification system, based on preoperative standing anteroposterior (AP) radiographs. All patients underwent full-leg standing AP radiographs preoperatively to analyze the HKA angle.

\section{Surgical Technique}

The patients were managed by three experienced surgeons who were familiar with the routine application of computer navigation in standard TKA practice. All surgeries were supervised by a single surgeon. The Stryker imageless navigation system (Precision version 4.0; Stryker Orthopedics, Mahwah, NJ), which does not require intraoperative fluoroscopy or preoperative computed tomography (CT) images, was used for computer-assisted implantation; the system used the kinematic acquisition of the hip center and anatomical acquisition of the knee and ankle centers.

After knee exposure using a parapatellar approach, active patient trackers were fixed onto the femur and tibia with two bicortical anchoring pins. Care was taken to avoid interfering with the knee motion; thus, pins were placed far from the surgical wound. Surgical exposure of the joint was performed to register the patient's anatomy according to standard procedure. After the registration of anatomical landmarks required for standard navigation workflows, the coronal plane alignment given by the femorotibial mechanical angle (FTMA) was recorded in extension in the neutral position without any applied stress or loading. Initially, no ligamentous release or osteophyte removal was performed, and the value was documented as the baseline mechanical alignment. The FTMA in maximum manual valgus stress (in an extended position) was recorded using computer navigation as a baseline measurement. These measurements were performed after partial closure of the arthrotomy. After anterior cruciate ligament sectioned, the subsequent two steps, defined as minimal medial soft-tissue release in this study, were then performed: (1) elevation of a periosteal sleeve to approximately $5-\mathrm{mm}$ distal to the joint line, which was circumferential to the entire tibiofemoral joint, including some deep medial collateral ligament $(\mathrm{MCL})$ fibers from the medial aspect of the tibia while retaining any remnants of the medial meniscus; and (2) complete removal of peripheral osteophytes from medial and posteromedial aspects of the proximal tibia and the distal medial femoral condyle. Specific attention was paid to the removal of all accessible osteophytes, and the tibia was subluxated anteriorly while having maximum external rotation torque applied to access osteophytes formation on the periphery of the posteromedial corner of the medial tibial plateau. The FTMA, both in neutral and in maximum manual valgus stress, was recorded using computer navigation after the two steps were performed. These measurements were performed after partial closure of the arthrotomy and were rounded off to the nearest 0.5 degrees. The posterior cruciate ligament was preserved in all cases until all measurements were completed. In knees with a flexion contracture, the measurements were performed at maximal extension. Once the data were obtained, the knee was opened again, and a standard TKA was performed. If the mechanical axis of the limb did not reach 
Table 1 Patient characteristics

\begin{tabular}{|l|l|}
\hline Age $^{\text {a }}$ & $76.4(63-96)$ \\
\hline sex & M: $32 \mathrm{F:} \mathrm{77}$ \\
\hline Affected side & R: 62 L: 69 \\
\hline HKA angle (degree) & 12.5 varus \pm 4.9 \\
\hline KL classification $^{\text {a }}$ & $3.8(3-4)$ \\
\hline Mean maximum extension (degree) & $-6.0 \pm 9.0$ \\
\hline Mean maximum flexion (degree) & $117 \pm 17.9$ \\
\hline
\end{tabular}

Abbreviations: F, female; HKA, hip-knee-ankle; KL, Kellgren-Lawrence; L, left; M, male; R, right.

${ }^{a}$ Values are expressed as numbers or mean (range) or median (range).

neutral on the navigation screen after implantation, additional procedures, such as medial release of the superficial MCL, medial tibial reduction osteotomy, or thin insert, were added on a case-by-case basis.

\section{Evaluation Criteria}

Preoperative variables, including age, sex, affected side, HKA angle, KL classification, maximum extension, and maximum flexion, were recorded. The evaluation criteria of this study included (1) the FTMA recorded after knee exposure in extension in the neutral position without any applied stress or loading which was defined as "the preoperative degree of varus deformity," (2) the FTMA after knee exposure in maximum manual valgus stress in an extended position, (3) the FTMA after minimum medial release in neutral position, and (4) the FTMA after minimum medial release in maximum manual valgus stress which was defined as "the mechanical angle with stress after medial release."

\section{Statistical Analysis}

Multivariate analysis was performed to examine the predictors of mechanical angle with stress after medial release. A gradient " $\mathrm{a} 1$ " and correlation coefficient " $r$ " were calculated by performing multiple linear regression analysis with " the mechanical angle with stress after medial release" as the dependent variable and "age," "height," "weight," "body mass index (BMI)," "sex," and "preoperative degree of varus deformity" as the independent variables. "Sex" was a dummy variable with male as 1 and female as 0 , and the optimal model was selected using the stepwise method. The multiple linear regressions between these criteria were analyzed to determine the threshold degree of varus deformity for obtaining neutral alignment after the two-step procedure. The level of statistical significance was set at $p=0.05$, and all calculations were performed using SPSS version 12 (SPSS Inc., Chicago, IL).

\section{Results}

Patient characteristics of 131 knees (109 patients) are shown in -Table 1. The mean age was 76.4 years (63-96) years, and there were 77 women and 32 men. The mean KL classification was 3.8, the HKA angle was $12.5 \pm 4.9$ degrees, and the flexion contracture was $6.0 \pm 9.0$ degrees.
The mean baseline mechanical alignment recorded in the neutral position before medial release (the preoperative degree of varus deformity) was $10.5 \pm 4.2$ degrees of varus (4-20.5 degrees) in extension. The mean degree of passive correctability with maximum valgus stress before intervention was $5.7+4.1$ degrees of varus (range: $0-20$ degrees). After soft-tissue release and osteophyte removal, passive correctability was increased to $0.7+2.6$ degrees of varus (-4.5-degree valgus to 10 -degree varus).

The results of multiple regression analysis between criteria are shown in - Table $\mathbf{2}$. The preoperative degree of varus deformity and sex were correlated with the mechanical angle with stress after medial release $(r=0.72, p<0.001)$. Age, height, weight, and BMI were not significantly correlated with the mechanical angle with stress after medial release. In the regression formula with the preoperative degree of varus and sex as independent variables, the regression coefficients of preoperative degree of varus deformity and male sex were $0.44(p<0.001)$ and $1.79(p<0.001)$, respectively. From this linear regression formula, the maximum degree of preoperative varus deformity that could obtain neutral alignment by a minimum medial-release procedure was calculated as 5.3 degrees for males and 9.1 degrees for females. The relationship between the preoperative degree of varus deformity and the mechanical angle with stress after medial release by sex is shown in - Fig. 1 .

\section{Discussion}

Although the release of deep MCL and osteophyte resection is routinely performed in TKA, ${ }^{17}$ minimal medial release should be performed if possible because excessive release of medial structures may lead to medial instability, midflexion instability, hematoma formation, knee joint elevation, need for constrained implants, and poor patient satisfaction. ${ }^{8,17,18}$ If a numerical guideline for the medial release can be established, optimal soft-tissue balance can be achieved with minimal medial release. This issue was less investigated in the past, although many surgeons have performed surgical procedures to obtain tissue balance, guided by their own experience. ${ }^{3,19,20}$ We found that the extent of limited joint line medial soft-tissue release and osteophyte removal to attain manual preresection deformity correction during navigation-assisted TKA 


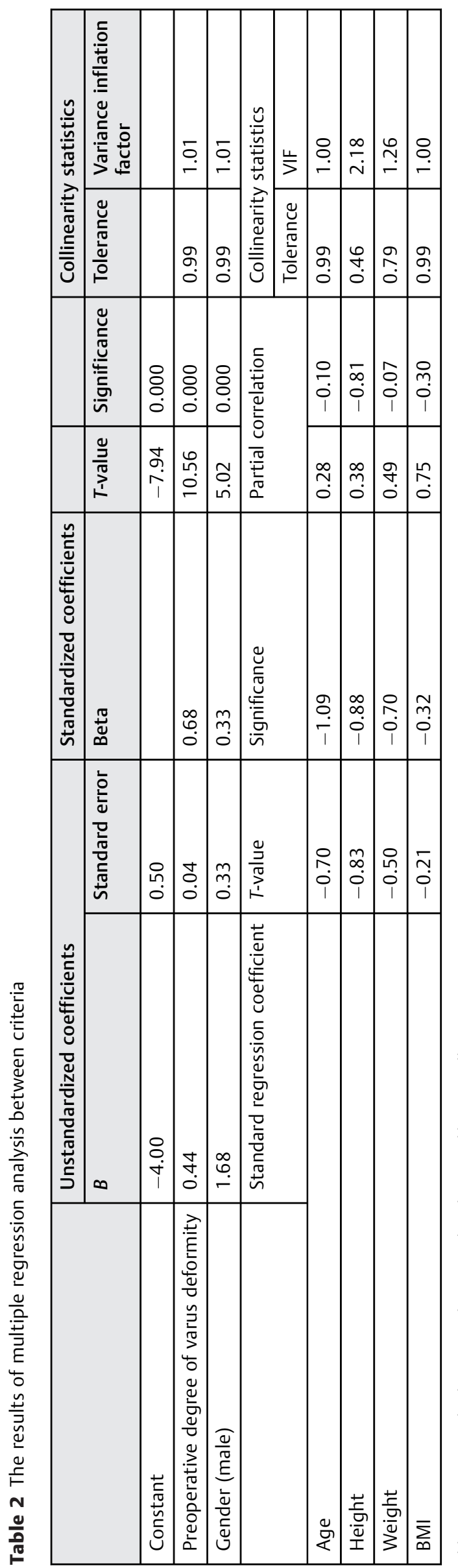

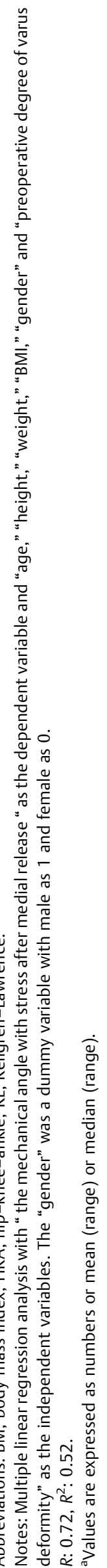

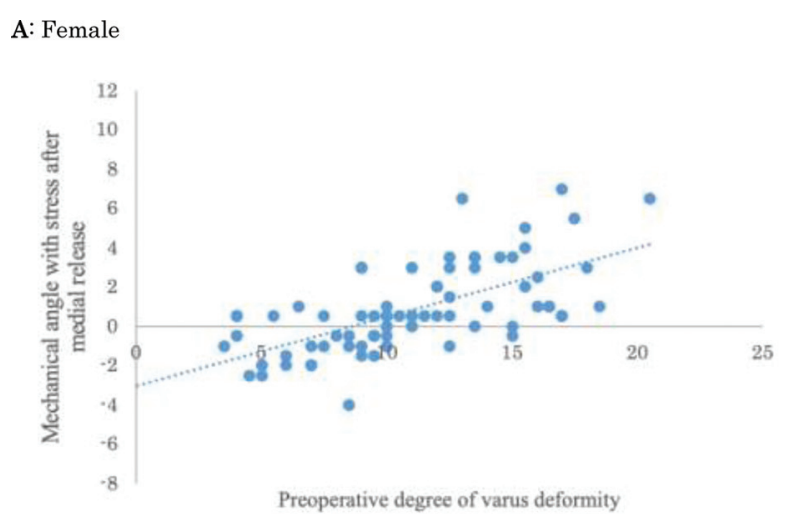

B: Male

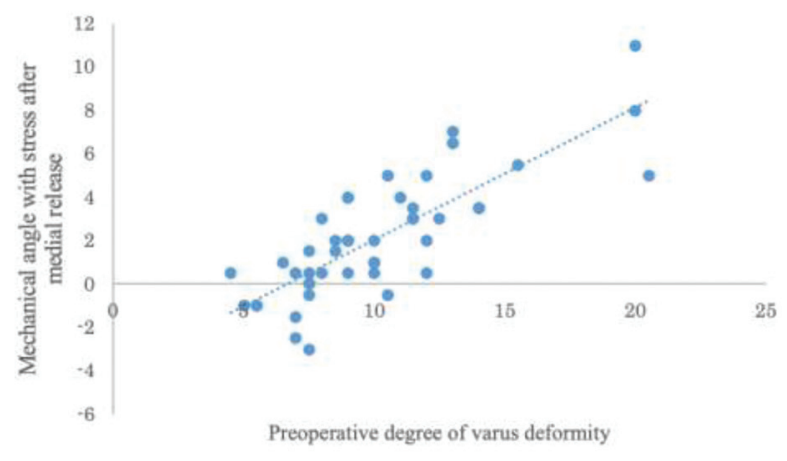

Fig. 1 The relationship between the preoperative degree of varus deformity and the mechanical angle with stress after medial release by gender. (A) Female. (B) Male.

procedures was 5.3 degrees for males and 9.1 degrees for females. If the preoperative degree of varus deformity was within this value, extensive medial release was not required to obtain neutral alignment according to the multiple regression analysis, which was based on the correlation between the degree of varus deformity preoperatively, sex, and after minimal medial release and complete osteophyte resection.

The main strength of this study was the large sample size. Previous studies that developed numerical guidelines on how to obtain soft-tissue balance with minimal medial release were performed by Picard et al and Bellemans et al as previously mentioned. ${ }^{21,22}$ These studies included 35 and 46 knees, respectively, whereas 131 knees were enrolled in our study. In addition, a numerical guideline for men and women were investigated separately in this present study. This allows for more specific and clinically relevant information. Furthermore, no specially designed protocols that included regular cruciate-retaining (CR) or posterior-stabilized (PS) TKA implants were applied, and every technique, including the measurement of the resection or gap balancing that affected the results of this study, was applicable.

Hakki et al reported from their data of 93 patients that the mechanical axis was restored before any bone resection other than osteophytes which led to achieving a stable, balanced TKA without collateral ligament release. ${ }^{23}$ This predictor showed a 
significant association with the need for collateral ligament release, with a sensitivity of $100 \%$ and specificity of $98 \%$. However, Hakki et al did not reveal the degree of varus deformity that could be corrected to neutral alignment with minimal medial release. The first study on a numerical guideline for medial release with navigation-assisted TKA was reported by Picard et al in 2007. ${ }^{21}$ Their navigation data indicated that not performing medial release (medial approach only) would allow 2-degree correction; a moderate release, such as MCL and semimembranosus, would allow 5 degrees, and an extensive release, such as posterior cruciate ligament and posterior capsule, would allow up to 8 degrees of correction. ${ }^{21}$ Nevertheless, their medial-release sequence was completely different from the procedure performed in this present study. Bellemans et al reported that knees with a varus deformity within 9 degrees were easily corrected to neutral after correction of extrinsic factors causing medial restriction such as osteophyte formation, adhesions to the underlying bone, and other factors. That study involved a similar technique and medial-release sequence to that of this study; they showed that there was a necessity for extensive release of the collateral ligament (because of the shortening of the medial collateral ligament) for a varus deformity greater than 10 degrees. ${ }^{4}$ However, these results were for both men and women. It has been previously reported that there are sex differences in knee joint laxity, and the magnitude of minimum medial release for correction of varus deformity is also expected to vary by sex. ${ }^{24,25}$ In practice, this study found that sex affected the mechanical angle with stress after medial release and the impact of sex difference in correction of varus deformity.

The benefit of this study is that we have demonstrated if the preoperative varus deformity angle is less than 5.3 degrees for males and 9.1 degrees for females, extensive medial release is needless, and the surgeon can proceed with the operation without anxiety. Once a normal medial soft-tissue envelope is achieved after performing the two steps in the varus TKA, a perfect soft-tissue balance may be achieved without the need for additional soft-tissue release. Similarly, the study shows how the superficial MCL and attachments of the pes anserinus and semimembranosus can be preserved, thereby achieving a well-functioning, pain-free knee joint without the risk of medial soft-tissue overrelease. Conversely, - Fig. 1 shows that if the preoperative varus deformity was more than 15 degrees in female and more than 10 degrees in male, it was difficult to reliably correct to neutral alignment with a limited release. In that case, additional medial release should be considered.

\section{Limitations and Strengths}

Our study had some limitations. First, this protocol relied on manual stress testing that is not quantitative. The interobserver agreement in the measurement of the mechanical angle in maximum manual valgus stress using intraclass correlation coefficients (ICCs) in this study was 0.88 . Even with confirmation using ICC, extensive experience, and repeated measurements during the procedure, the data values may vary. Second, data were obtained from a comput- er software source, and we are unsure of the reproducibility of these using other software or if a comparable software is available to measure the alignment in real-time. Third, this study did not evaluate the duration of osteoarthritis in the patients treated. It is possible that chronicity of the arthritic process might impact soft-tissue compliance and the response to deformity correction with a limited release. Fourth, we did not incorporate posterior femoral osteophyte removal or menisectomy into our release. From a previous study by Sriphirom et al, removal of the posterior condylar osteophyte could correct a varus deformity by $0.90 \pm 1.14$ degrees. ${ }^{2627}$ Therefore, if the preoperative varus deformity was within 6.2 degrees for males and 10.0 degrees for females, minimal medial release and resection of osteophytes allowing for correction of approximately 5.3 degrees for males and 9.1 degrees for females (based on this present study) and posterior clearance allowing for an additional correction of 0.9 degrees (based on the study by Sriphirom et al), will allow for correction to neutral alignment. ${ }^{26}$ Fifth, we did not evaluate the size of osteophytes. If there were large osteophytes preoperatively, the osteophyte resection may greatly correct the varus deformation.

Despite these limitations, the study results may provide a framework for surgeons to use when considering the initial medial release that they will perform for patients with varus alignment less than 5.3 degrees for males and 9.1 degrees for females.

\section{Conclusion}

The extent for limited joint line medial soft-tissue release and osteophyte removal to attain manual preresection deformity correction during navigation-assisted TKA procedures was 5.3 degrees for males and 9.1 degrees for females. If the preoperative degree of varus deformity was within 5.3 degrees for males and 9.1 degrees for females, extensive medial release was not required to obtain neutral alignment.

Funding

None.

Conflict of Interest

None declared.

\section{References}

1 Unitt L, Sambatakakis A, Johnstone D, Briggs TW; Balancer Study Group. Short-term outcome in total knee replacement after softtissue release and balancing. J Bone Joint Surg Br 2008;90(02): 159-165

2 Whiteside LA, Saeki K, Mihalko WM. Functional medical ligament balancing in total knee arthroplasty. Clin Orthop Relat Res 2000; (380):45-57

3 Aunan E, Kibsgård T, Clarke-Jenssen J, Röhrl SM. A new method to measure ligament balancing in total knee arthroplasty: laxity measurements in 100 knees. Arch Orthop Trauma Surg 2012;132 (08):1173-1181

4 Babazadeh S, Stoney JD, Lim K, Choong PF. The relevance of ligament balancing in total knee arthroplasty: how important 
is it? A systematic review of the literature. Orthop Rev (Pavia) 2009;1(02):e26

5 King JJ III, Chakravarty R, Cerynik DL, Black A, Johanson NA. Decreased ratios of lateral to medial patellofemoral forces and pressures after lateral retinacular release and gender knees in total knee arthroplasty. Knee Surg Sports Traumatol Arthrosc 2013;21(12):2770-2778

6 Sikorski JM. Alignment in total knee replacement. J Bone Joint Surg Br 2008;90(09):1121-1127

7 Azukizawa M, Kuriyama S, Nakamura S, et al. Intraoperative medial joint laxity in flexion decreases patient satisfaction after total knee arthroplasty. Arch Orthop Trauma Surg 2018;138(08):1143-1150

8 Ramappa M. Midflexion instability in primary total knee replacement: a review. SICOT J 2015;1:24

9 Tsukiyama H, Kuriyama S, Kobayashi M, et al. Medial rather than lateral knee instability correlates with inferior patient satisfaction and knee function after total knee arthroplasty. Knee 2017; 24(06):1478-1484

10 Rossi R, Cottino U, Bruzzone M, Dettoni F, Bonasia DE, Rosso F. Total knee arthroplasty in the varus knee: tips and tricks. Int Orthop 2019;43(01):151-158

11 Völlner F, Fischer J, Weber M, et al. Weakening of the knee ligament complex due to sequential medial release in total knee arthroplasty. Arch Orthop Trauma Surg 2019;139(07): 999-1006

12 Wada K, Hamada D, Tamaki S, Higashino K, Fukui Y, Sairyo K. Influence of medial collateral ligament release for internal rotation of tibia in posterior-stabilized total knee arthroplasty: a cadaveric study. J Arthroplasty 2017;32(01):270-273

13 McClelland JA, Webster KE, Ramteke AA, Feller JA. Total knee arthroplasty with computer-assisted navigation more closely replicates normal knee biomechanics than conventional surgery. Knee 2017;24(03):651-656

14 Picard F, Deep K, Jenny JY. Current state of the art in total knee arthroplasty computer navigation. Knee Surg Sports Traumatol Arthrosc 2016;24(11):3565-3574

15 Kawata M, Sasabuchi Y, Inui H, et al. Annual trends in knee arthroplasty and tibial osteotomy: analysis of a national database in Japan. Knee 2017;24(05):1198-1205
16 Maradit Kremers H, Larson DR, Crowson CS, et al. Prevalence of total hip and knee replacement in the United States. J Bone Joint Surg Am 2015;97(17):1386-1397

17 Koh HS, In Y. Semimembranosus release as the second step of soft tissue balancing in varus total knee arthroplasty. J Arthroplasty 2013;28(02):273-278

18 Athwal KK, Daou HE, Kittl C, Davies AJ, Deehan DJ, Amis AA. The superficial medial collateral ligament is the primary medial restraint to knee laxity after cruciate-retaining or posteriorstabilised total knee arthroplasty: effects of implant type and partial release. Knee Surg Sports Traumatol Arthrosc 2016;24 (08):2646-2655

19 Elmallah RK, Mistry JB, Cherian JJ, et al. Can we really "feel" a balanced total knee arthroplasty? J Arthroplasty 2016;31(9, suppl):102-105

20 Gustke K. Use of smart trials for soft-tissue balancing in total knee replacement surgery. J Bone Joint Surg Br 2012;94(11, suppl A): $147-150$

21 Picard F, Deakin AH, Clarke IV, Dillon JM, Kinninmonth AW. A quantitative method of effective soft tissue management for varus knees in total knee replacement surgery using navigational techniques. Proc Inst Mech Eng H 2007;221(07):763-772

22 Bellemans J, Vandenneucker H, Vanlauwe J, Victor J. The influence of coronal plane deformity on mediolateral ligament status: an observational study in varus knees. Knee Surg Sports Traumatol Arthrosc 2010;18(02):152-156

23 Hakki S, Coleman S, Saleh K, Bilotta VJ, Hakki A. Navigational predictors in determining the necessity for collateral ligament release in total knee replacement. J Bone Joint Surg Br 2009;91 (09):1178-1182

24 Boguszewski DV, Cheung EC, Joshi NB, Markolf KL, McAllister DR. Male-female differences in knee laxity and stiffness. Am J Sports Med 2015;43(12):2982-2987

25 Deep K. Collateral ligament laxity in knees: what is normal? Clin Orthop Relat Res 2014;472(11):3426-3431

26 Sriphirom P, Siramanakul C, Chanopas B, Boonruksa S. Effects of posterior condylar osteophytes on gap balancing in computerassisted total knee arthroplasty with posterior cruciate ligament sacrifice. Eur J Orthop Surg Traumatol 2018;28(04):677-681 